\title{
The large-leaved Kudingcha (Ilex latifolia Thunb and Ilex kudingcha C.J. Tseng): a traditional Chinese tea with plentiful secondary metabolites and potential biological activities
}

\author{
Li Li $\cdot$ Li J. Xu • Gui Z. Ma $\cdot$ Yin M. Dong $\cdot$ \\ Yong Peng $\cdot$ Pei G. Xiao
}

Received: 21 October 2012/ Accepted: 20 February 2013/Published online: 26 March 2013

(C) The Japanese Society of Pharmacognosy and Springer Japan 2013

\begin{abstract}
In China, Kudingcha has been used for almost 2,000 years as a tea to quench thirst, remove phlegm, refresh the mind, and improve eyesight. The group of large-leaved Kudingcha is coveted for its potential effects on lipid metabolism, which are attributed to the presence of characteristic ingredients. This contribution reviews studies from the past few decades regarding the plant characteristics, ethnobotanical usages, chemical constituents, and related biological activities of the large-leaved Kudingcha (Ilex latifolia Thunb and Ilex kudingcha C.J. Tseng). Triterpenoids, phenolic acids, flavonoids, and essential oils are the main metabolites in the large-leaved Kudingcha, and these ingredients protect the vascular system, regulate lipid metabolism, and have antioxidant, hypoglycemic, and anti-tumor effects. Moreover, large-leaved Kudingcha shares several properties with the popular green tea and the Yerba maté from South America.
\end{abstract}

Keywords Large-leaved Kudingcha - Ilex . Plant characteristics - Ethnobotanical usages . Chemical constituents - Therapeutic properties . Yerba maté

L. Li (凹) · Y. M. Dong

Beijing Key Laboratory of Plant Resources Research and Development, Beijing Technology and Business University, Beijing 100048, China e-mail: lily002006@yahoo.com.cn

L. J. Xu · Y. Peng · P. G. Xiao

Institute of Medicinal Plant Development, Chinese Academy of Medical Sciences, Beijing 100193, China

G. Z. Ma

College of Pharmacy, Xinjiang Medical University,

Urumqi 830011, Xinjiang, China

\section{Introduction}

Kudingcha is a particularly bitter-tasting tea that has been widely used in China throughout history. It is known to disperse wind-heat, clear toxins from the blood, and refresh the mentalities. It has also been used to cure the common cold, rhinitis, itching eyes, conjunctival congestion, and headache; it is helpful for digestion and alleviating the adverse effects of alcohol [1,2]. Today, nearly 20 plants from different families with similarities in appearance, flavor, and traditional usage in different areas of China are all named "Kudingcha" [3]. However, the plants most commonly found in the markets of China can be divided into 2 groups: one group, including species Ilex latifolia Thunb and I. kudingcha C.J. Tseng, was named the "largeleaved Kudingcha"; the other group, containing the species Ligustrum robustum (syn. Ligustrum purpurascens), was named the "small-leaved Kudingcha." The large-leaved Kudingcha was certified to be the original Kudingcha species and has obvious antioxidant, anti-inflammatory, lipid metabolism, hepatoprotective, and anti-tumor activities [4-7], similar to the popular green tea (Camellia sinensis) and the Yerba maté tea (Ilex paraguariensis) from South America. Over the last several years, Kudingcha has been considered as a dietetic beverage and is gaining popularity with names like "beauty-slimming tea," "longevity tea," "green-golden tea," and "clearing-heat tea."

In the current review, we present and analyze the plant characteristics, ethnobotanical usages, phytochemistry, and pharmacological activities of the large-leaved Kudingcha, as well as its relationship with green tea and Yerba maté tea. These up-to-date research observations will be helpful in understanding the characteristics and superiorities of this special tea and would be applicable in developing new products and herbal medicines in the future. 


\section{Historical and ethnopharmacological investigation of Kudingcha in China}

In the book “Tong Jun Lu” (桐君录) (A.D. 25-225; E. Han Dynasty), Kudingcha was described as having a bitter flavor and reportedly kept people awake all night [8]. The original species in "Tong Jun Lu" were investigated and certified to be I. latifolia, I. kudingcha, and I. cortia [9]. At present, I. cortia is called "Gougucha" and is classified separately from Kudingcha due to its different botanical characters, chemical ingredients, and medicinal usages [11]. On the basis of our investigation of the literature, plant resources, and chemical and molecular analyses conducted since 2006, it appears that the large-leaved Kudingcha from the genus Ilex (I. latifolia and I. kudingcha) is the original Kudingcha species [2, 8, 11].

The medicinal properties of Kudingcha were reported in “Ben Cao Shi Yi” (本草拾遗) of the Tang Dynasty, including clearing the thirst and phlegm, resolving hydropsia, refreshing the mind, and improving the eyesight. In the encyclopedia "Ben Cao Gang Mu" (本草纲目) of the Ming Dynasty, Kudingcha was described as an effective herb that contained no toxins and had medicinal properties that could scatter wind-heat, refresh the mind and spirit, and promote fluid production to quench thirst [3]. The records in ancient books were all about the large-leaved Kudingcha from the genus Ilex. The current application of I. latifolia is limited to a few provinces of Eastern China. The supply and market share are limited compared to that of I. kudingcha.

The other important group (the small-leaved Kudingcha) from the genus Ligustrum was first reported in the book of "Guizhou Min Jian Fang Yao Ji" in 1958. Some investigators put forward that the small-leaved Kudingcha originated from the mountains of Dalou and the river valley of
Wujiang in Guizhou province. It has been used in the civilian Tujia ethnic group and Miao ethnic group for a long time, so was named as one of the four famous old tea species locally $[2,10]$. No literal records have been found in ancient books to date. The small-leaved Kudingcha ( $L$. robustum) was initially used because of its similarities in plant morphology, bitter flavor, and ethnobotanical use in folk medicine.

\section{Plant characteristics and distribution of Kudingcha species}

Two species of the large-leaved Kudingcha from the genus Ilex have very similar botanical appearances. Both come from trees that are evergreen, and the primary difference between the species is found at the base of the leaves [4]. The leaf characteristics of Kudingcha species are listed in Table 1. The small-leaved Kudingcha from the genus Ligustrum are shrubs or small trees, deciduous or evergreen. The leaves are much smaller and thinner than the large-leaved Kudingcha, and the flavor is not as bitter as, which makes it more suitable to drink routinely [8]. According to Table 1, the essential leaf characteristics of each species are useful to distinguish Kudingcha original plants. While these Kudingcha species are distributed across different locations over China, they have similar ethnopharmacological properties (Table 2).

\section{Secondary metabolites of large-leaved Kudingcha}

Triterpenoids, phenolic acids, flavonoids, and essential oils were isolated and identified from the large-leaved

Table 1 Leaf characteristics of the most commonly used Kudingcha [65, 66]

\begin{tabular}{lllc}
\hline $\begin{array}{l}\text { Leaf } \\
\text { characteristics }\end{array}$ & I. kudingcha & I. latifolia & L. robustum \\
\hline Blade & Oblong to oblong-elliptic & Oblong or ovate oblong & $\begin{array}{c}\text { Lanceolate to subovate } \\
\text { or elliptic }\end{array}$ \\
Petiole & $\begin{array}{l}2-2.2 \mathrm{~cm}, \text { abaxially subrounded, rugose, adaxially sulcate, } \\
\text { puberulent }\end{array}$ & $\begin{array}{c}\text { Subterete, abaxially rugose, adaxially } \\
\text { slightly impressed }\end{array}$ & $\begin{array}{c}2-8 \text { mm, pubescent, } \\
\text { groovy }\end{array}$ \\
Margin & Doubly serrate or densely serrate & Sparsely serrate & - \\
Base & Obtuse or cuneate & Rounded or broadly cuneate & Broadly cuneate or \\
& & & subrounded \\
Apex & Acute or short acuminate & Short or long acuminate & Long acuminate \\
Texture & Leathery & Thickly leathery & Papery \\
Mid vein & Raised and keeled abaxially, impressed and sparsely & Impressed adaxially & - \\
Lateral vein & Eviderulent adaxially & & - \\
Vein-pair & $5-7$ & Obscure abaxially, obvious adaxially & - \\
Vein-angle & About $50^{\circ}$ & - & - \\
\hline
\end{tabular}


Table 2 Distribution and medical uses of the most commonly used Kudingcha

\begin{tabular}{|c|c|c|c|}
\hline Common name & Species & Distribution & Recorded uses [8] \\
\hline \multirow[t]{2}{*}{ Large-leaved Kudingcha } & I. kudingcha & Guangxi, Guangdong, Hannan, Hunan & $\begin{array}{l}\text { Clearing pathogenic summer-heat and } \\
\text { detoxifying, treatment of the } \\
\text { dehydration and abdominal pain }\end{array}$ \\
\hline & I. latifolia & Zhejiang, Jiangxi, Jiangsu & $\begin{array}{l}\text { Removing the excessive fire and noxious } \\
\text { heat from the lung and spleen, removing } \\
\text { the damp-heat pathogens in the large } \\
\text { intestine to eliminate the dysentery, } \\
\text { mental refreshing }\end{array}$ \\
\hline Small-leaved Kudingcha & $\begin{array}{l}\text { L. robustum } \\
\quad \text { (syn. L. purpurascens) }\end{array}$ & Guizhou, Yunnan, Sichuan & $\begin{array}{l}\text { Mental refreshing, treatment of the } \\
\text { pathogenic summer-heat, polydipsia and } \\
\text { thirst, headache, conjunctival } \\
\text { congestion }\end{array}$ \\
\hline
\end{tabular}

Kudingcha. Among different types of constituents, triterpenoids and polyphenols were considered to be the most important metabolites, with various bioactivities (summarized in Fig. 1).

\section{Triterpenoids}

The ursane-type triterpenoids with lactone at the position of C20 and C28 are called $\alpha$-kudinlactone $(48-57,113)$, $\beta$-kudinlactone (58-69), and $\gamma$-kudinlactone (74-78) (Fig. 1), and are considered to be the most characteristic chemicals in the Kudingcha species. The chemical analysis showed that I. kudingcha had the highest total triterpenoid content. The $\beta$-kudinlactone was the main type of triterpenoid present in I. latifolia, while $\gamma$-kudinlactone and $\alpha$-kudinlactone were not present in I. latifolia. At the same time, none of these characteristic kudinosides were detected in the supposititious species I. pentagona and I. cornuta of China, and the similar Yerba maté of South America [11]. In addition, oleanane-type (1-13) and lupane-type triterpenes (81-87) and their glycosides were also found in I. latifolia and I. kudingcha.

\section{Phenolic acids}

Phenolic acids with antioxidant activities have been found in recent years. The main types of phenolic acids found in large-leaved Kudingcha were polyphenols and related compounds [30, 32]. High-performance liquid chromatography (HPLC) analysis showed the presence of 6 of these derivatives, but no caffeine, in female and male leaves of I. latifolia. However, the levels of these derivatives varied from season to season. The current-year spring leaves showed the highest levels of caffeic acid derivatives (147-235 mg/g), while the current-year autumn leaves, old spring leaves, and old autumn leaves had lower amounts of the derivatives of $36,24-44$, and $31 \mathrm{mg} / \mathrm{g}$, respectively. The levels of 4-O-caffeoyl quinic acid increased 3-6 times in current-year leaves from spring to autumn. The contents of the caffeic acid derivatives in the female plant were similar to those in the old leaves of the male plant. These results suggest that the new spring leaves of I. latifolia would be a good source for antioxidants [33].

Flavonoids

Compounds 90-92 and 119-121 were the main flavones isolated from the large-leaved Kudingcha. They are also the primary constituents in the popular green tea.

Essential oils and other secondary metabolites

The essential oils have been studied by using gas chromatography coupled to mass spectrometry (GC/MS), which indicated the presence of alcohol, aldehyde, ketone, ether, fatty acids, and fatty acid ester [34, 35]. Other secondary metabolites such as phytosterols and polysaccharides were also isolated and identified [26, 41].

\section{Biological activities}

Regularly drinking Kudingcha as a herbal tea has a positive role in the prevention and treatment of cardiovascular and cerebrovascular diseases, diabetes, pharyngitis, and cancer, particularly for the relevant troublesome conditions of arteriosclerosis, hypertension, dizziness, insomnia, palpitations, and chest tightness caused by cardiovascular diseases [8]. The most important biological activity of the tea is lipid metabolism activity. Moreover, it has antioxidant and antitumor activities, and has effects on the hypoglycemic and vascular system, similar to green tea and Yerba maté [2].

Lipid metabolism activity

An acyl CoA cholesteryl acyl transferase (ACAT) catalyzes the intracellular esterification of cholesterol in various tissues, and inhibitors of ACAT may serve as new 

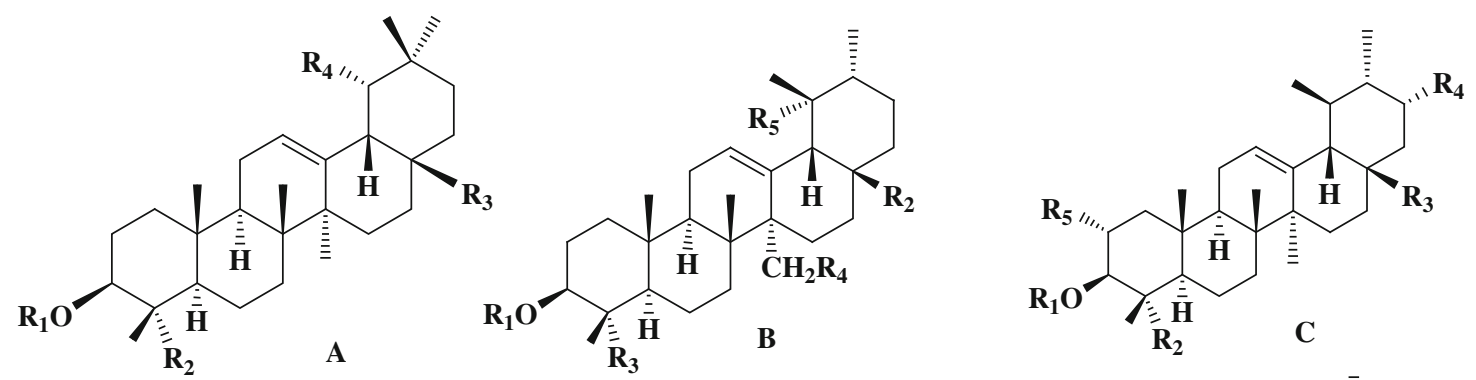

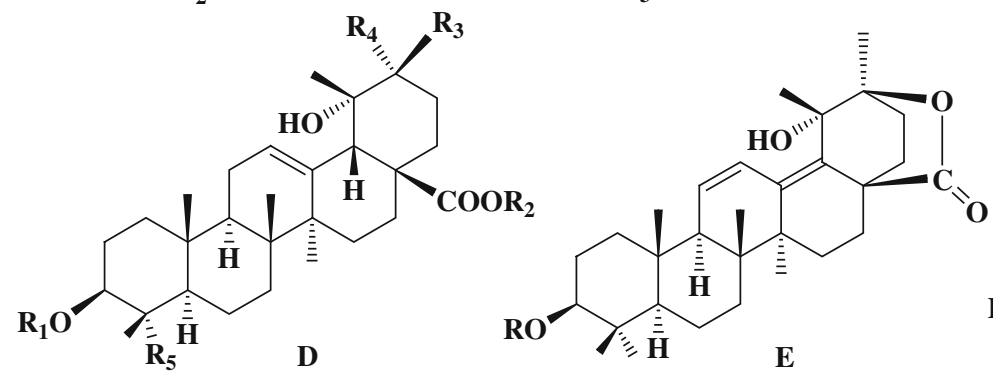

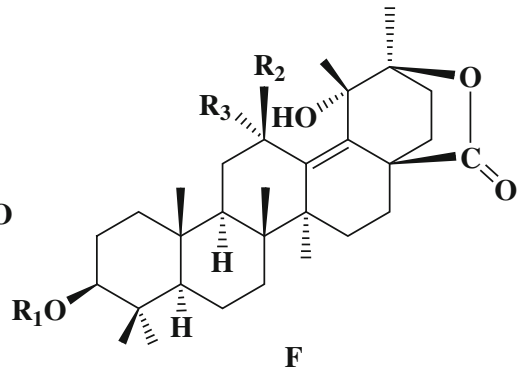

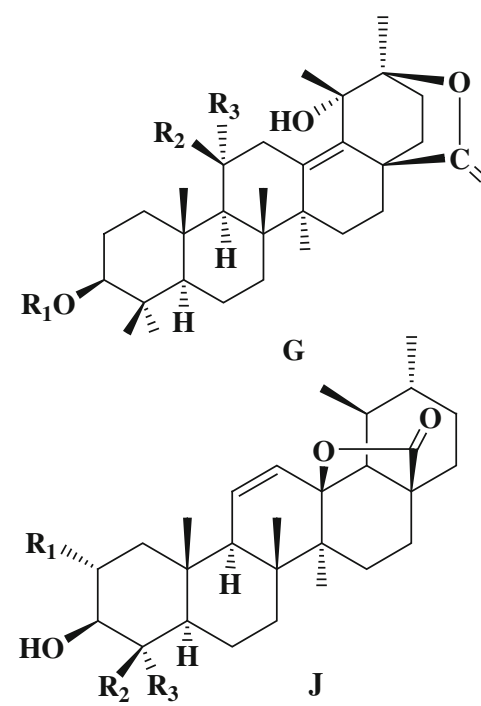

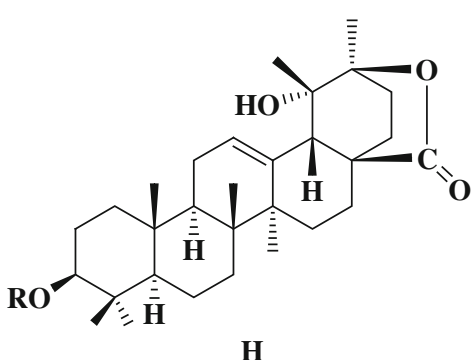

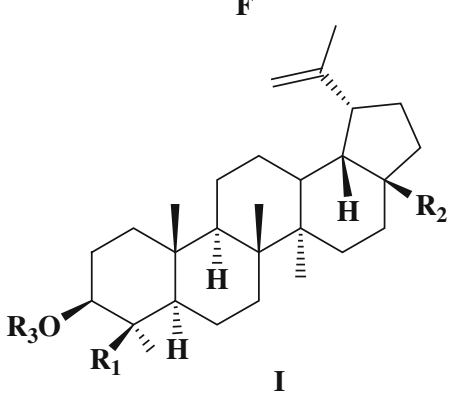<smiles>[R4]c1cc(-c2oc3cc(O)cc(O)c3c(=O)c2OCC)ccc1O</smiles>

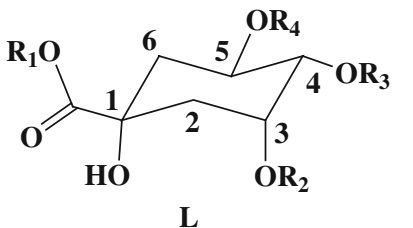

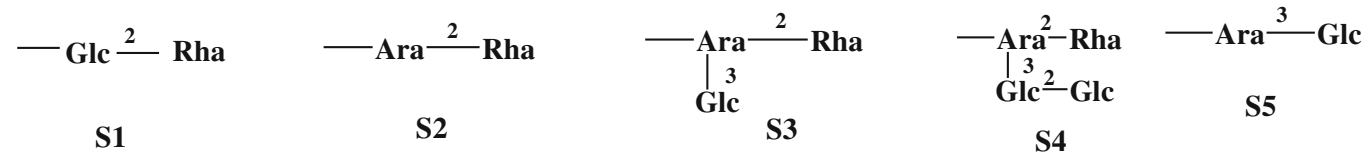

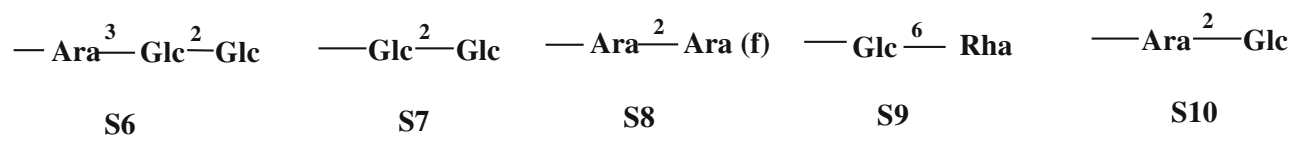<smiles>CC(=O)/C=C/c1ccc(O)c(O)c1</smiles>

O-E-p-coumaroyl

01

\section{O-Z-p-coumaroyl}

$\mathrm{O2}$

Fig. 1 The chemical structures of compounds isolated from the large-leaved Kudingcha 


\begin{tabular}{|c|c|c|c|c|c|c|c|c|}
\hline No. & Compound name & $\begin{array}{l}\text { Skel } \\
\text { eton } \\
\text { e }\end{array}$ & $\mathbf{R} 1$ & $\mathbf{R} 2$ & $\mathbf{R 3}$ & R4 & $\mathbf{R 5}$ & Ref \\
\hline 1 & $\beta$-amyrin & A & $\mathrm{H}$ & $\mathrm{Me}$ & $\mathrm{Me}$ & $\mathrm{H}$ & - & [22] \\
\hline 2 & erythrodiol & A & $\mathrm{H}$ & $\mathrm{Me}$ & $\mathrm{CH}_{2} \mathrm{OH}$ & $\mathrm{H}$ & - & [22] \\
\hline 3 & oleanolic acid & A & $\mathrm{H}$ & $\mathrm{Me}$ & $\mathrm{COOH}$ & $\mathrm{H}$ & - & [22] \\
\hline 4 & ilexosideXL VIII & A & GlcA & $\mathrm{CH}_{2} \mathrm{OH}$ & COOGlc & $\mathrm{H}$ & - & [6] \\
\hline 5 & ilekudinoside A (latifoloside O) & A & $\mathrm{S} 3$ & $\mathrm{Me}$ & COOGlc & $\mathrm{H}$ & - & {$[6,18]$} \\
\hline 6 & mateglycoside D & A & $\mathrm{S} 2$ & $\mathrm{Me}$ & COOGlc & $\mathrm{H}$ & - & [26] \\
\hline 7 & latifoloside P & A & $\mathrm{S} 2$ & $\mathrm{Me}$ & COOS1 & $\mathrm{H}$ & - & {$[18]$} \\
\hline 8 & latifoloside Q & A & S3 & $\mathrm{Me}$ & COOS1 & $\mathrm{H}$ & - & {$[18]$} \\
\hline 9 & latifoloside B & A & $\mathrm{S} 2$ & $\mathrm{Me}$ & COOGlc & $\mathrm{OH}$ & - & {$[15]$} \\
\hline 10 & latifoloside $\mathrm{C}$ & $\mathrm{A}$ & $\mathrm{S} 3$ & $\mathrm{Me}$ & COOGlc & $\mathrm{OH}$ & - & {$[15]$} \\
\hline 11 & kudinoside $\mathrm{N}$ & A & S4 & $\mathrm{Me}$ & COOGlc & $\mathrm{OH}$ & - & [19] \\
\hline 12 & kudinoside $\mathrm{O}$ & A & S4 & $\mathrm{Me}$ & COOS1 & $\mathrm{OH}$ & - & [19] \\
\hline 13 & latifoloside $\mathrm{H}$ & A & S3 & $\mathrm{Me}$ & COOS1 & $\mathrm{OH}$ & - & [17] \\
\hline 14 & $\alpha$-amyrin & B & $\mathrm{H}$ & $\mathrm{Me}$ & $\mathrm{Me}$ & $\mathrm{H}$ & $\mathrm{H}$ & [22] \\
\hline 15 & ursolic acid 3-O-acetate & $\mathrm{B}$ & acetyl & $\mathrm{COOH}$ & $\mathrm{Me}$ & $\mathrm{H}$ & $\mathrm{H}$ & [22] \\
\hline 16 & uvaol & $\mathrm{B}$ & $\mathrm{H}$ & $\mathrm{CH}_{2} \mathrm{OH}$ & $\mathrm{Me}$ & $\mathrm{H}$ & $\mathrm{H}$ & [22] \\
\hline 17 & uvaol 3-O-acetate & B & acetyl & $\mathrm{CH}_{2} \mathrm{OH}$ & $\mathrm{Me}$ & $\mathrm{H}$ & $\mathrm{H}$ & [22] \\
\hline 18 & ursolic acid & B & $\mathrm{H}$ & $\mathrm{COOH}$ & $\mathrm{Me}$ & $\mathrm{H}$ & $\mathrm{H}$ & [22] \\
\hline 19 & $3 \beta$ - palmitoyl- $\alpha$-amyrin & $\mathrm{B}$ & $\begin{array}{l}\mathrm{CH}_{3}\left(\mathrm{CH}_{2}\right)_{14} \\
\mathrm{CO}\end{array}$ & $\mathrm{Me}$ & $\mathrm{Me}$ & $\mathrm{H}$ & $\mathrm{H}$ & {$[16]$} \\
\hline 20 & 23-hydroxyursolic acid & $\mathrm{B}$ & $\mathrm{H}$ & $\mathrm{COOH}$ & $\mathrm{CH}_{2} \mathrm{OH}$ & $\mathrm{H}$ & $\mathrm{H}$ & [5] \\
\hline 21 & 27-trans-p-coumaroyloxyursolic acid & $\mathrm{B}$ & $\mathrm{H}$ & $\mathrm{COOH}$ & $\mathrm{Me}$ & O1 & $\mathrm{H}$ & [5] \\
\hline 22 & 27-cis-p-coumaroyloxyursolic acid & $\mathrm{B}$ & $\mathrm{H}$ & $\mathrm{COOH}$ & $\mathrm{CH}_{2} \mathrm{OH}$ & $\mathrm{O} 2$ & $\mathrm{H}$ & [5] \\
\hline 23 & ilekudinoside B & $\mathrm{B}$ & GlcA & COOGlc & $\mathrm{Me}$ & $\mathrm{H}$ & $\mathrm{OH}$ & [6] \\
\hline 24 & ilekudinoside D & B & Ara & COOGlc & $\mathrm{CH}_{2} \mathrm{OH}$ & $\mathrm{H}$ & $\mathrm{OH}$ & [6] \\
\hline 25 & ilekudinoside E (latifoloside L) & B & S5 & COOGlc & $\mathrm{Me}$ & $\mathrm{H}$ & $\mathrm{OH}$ & {$[6,18]$} \\
\hline 26 & kudinoside $\mathrm{H}$ & B & Ara & COOGlc & $\mathrm{Me}$ & $\mathrm{H}$ & $\mathrm{OH}$ & [14] \\
\hline 27 & kudinoside G & B & S3 & COOGlc & $\mathrm{Me}$ & $\mathrm{H}$ & $\mathrm{OH}$ & [14] \\
\hline 28 & kudinoside L & B & S4 & COOGlc & $\mathrm{Me}$ & $\mathrm{H}$ & $\mathrm{OH}$ & [19] \\
\hline 29 & kudinoside P & $\mathrm{B}$ & S6 & COOGlc & $\mathrm{CH}_{2} \mathrm{OH}$ & $\mathrm{H}$ & $\mathrm{OH}$ & [19] \\
\hline 30 & kudinoside M & $\mathrm{B}$ & $\mathrm{S} 4$ & coos1 & $\mathrm{Me}$ & $\mathrm{H}$ & $\mathrm{OH}$ & [19] \\
\hline 31 & latifoloside G & B & S3 & coos1 & $\mathrm{Me}$ & $\mathrm{H}$ & $\mathrm{OH}$ & [17] \\
\hline 32 & ilekudinoside W & B & $\mathrm{S} 2$ & COOS1 & $\mathrm{Me}$ & $\mathrm{H}$ & $\mathrm{OH}$ & [24] \\
\hline 33 & latifoloside A & $\mathrm{B}$ & $\mathrm{S} 2$ & COOGlc & $\mathrm{Me}$ & $\mathrm{H}$ & $\mathrm{OH}$ & {$[15]$} \\
\hline 34 & latifoloside I (1) & $\mathrm{C}$ & S1 & $\mathrm{Me}$ & $\mathrm{CH}_{2} \mathrm{OH}$ & OGlc & $\mathrm{H}$ & [23] \\
\hline 35 & latifoloside J (1) & $\mathrm{C}$ & S1 & $\mathrm{Me}$ & $\mathrm{COOH}$ & OGlc & $\mathrm{H}$ & [23] \\
\hline 36 & cynarasaponin $\mathrm{C}$ & $\mathrm{C}$ & Glc A & $\mathrm{CH}_{2} \mathrm{OH}$ & COOGlc & $\mathrm{H}$ & $\mathrm{H}$ & [6] \\
\hline 37 & ilekudinoside C & $\mathrm{C}$ & Ara & $\mathrm{CH}_{2} \mathrm{OH}$ & COOGlc & $\mathrm{H}$ & $\mathrm{OH}$ & {$[6]$} \\
\hline 38 & $\begin{array}{l}\text { 3- } \beta \text {-D-glucuronopyranosyl asiatic acid } \\
28-\mathrm{O}-\beta \text {-D-glucopyranoside }\end{array}$ & $\mathrm{C}$ & Glc A & $\mathrm{CH}_{2} \mathrm{OH}$ & COOGlc & $\mathrm{H}$ & $\mathrm{OH}$ & [19] \\
\hline
\end{tabular}

Fig. 1 continued 


\begin{tabular}{|c|c|c|c|c|c|c|c|c|}
\hline 39 & kudinoside I (1) & $\mathrm{D}$ & $\mathrm{S} 3$ & S1 & $\mathrm{OH}$ & $\mathrm{Me}$ & $\mathrm{Me}$ & [19] \\
\hline 40 & kudinoside $\mathrm{J}$ (1) & $\mathrm{D}$ & S4 & Glc & $\mathrm{OH}$ & $\mathrm{Me}$ & $\mathrm{Me}$ & [19] \\
\hline 41 & kudinoside $\mathrm{K}$ & $\mathrm{D}$ & S4 & S1 & $\mathrm{OH}$ & $\mathrm{Me}$ & $\mathrm{Me}$ & [19] \\
\hline 42 & latifoloside I (2) & $\mathrm{D}$ & S5 & Glc & $\mathrm{Me}$ & $\mathrm{H}$ & $\mathrm{Me}$ & [18] \\
\hline 43 & latifoloside $\mathbf{J}$ (2) & $\mathrm{D}$ & S2 & $\mathrm{S} 1$ & $\mathrm{Me}$ & $\mathrm{H}$ & $\mathrm{Me}$ & [18] \\
\hline 44 & latifoloside K & $\mathrm{D}$ & S5 & Glc & $\mathrm{Me}$ & $\mathrm{H}$ & $\mathrm{CH}_{2} \mathrm{OH}$ & [18] \\
\hline 45 & latifoloside $\mathrm{C}$ & $\mathrm{D}$ & S2 & Glc & $\mathrm{Me}$ & $\mathrm{H}$ & $\mathrm{Me}$ & [15] \\
\hline 46 & latifoloside D & $\mathrm{D}$ & S3 & Glc & $\mathrm{Me}$ & $\mathrm{H}$ & $\mathrm{Me}$ & [15] \\
\hline 47 & latifoloside F & $\mathrm{D}$ & S3 & S1 & $\mathrm{Me}$ & $\mathrm{H}$ & $\mathrm{Me}$ & [17] \\
\hline 48 & $\alpha$-kudinlactone & $\mathrm{E}$ & $\mathrm{H}$ & - & - & - & - & [14] \\
\hline 49 & kudinoside D & $\mathrm{E}$ & $\mathrm{S} 3$ & - & - & - & - & [14] \\
\hline 50 & kudinoside E & $\mathrm{E}$ & S4 & - & - & - & - & [14] \\
\hline 51 & kudinoside $\mathbf{J}$ (2) & $\mathrm{E}$ & Ara & - & - & - & - & [14] \\
\hline 52 & ilekudinoside K & $\mathrm{E}$ & S1 & - & - & - & - & [20] \\
\hline 53 & ilekudinoside $\mathrm{N}$ & $\mathrm{E}$ & S7 & - & - & - & - & [20] \\
\hline 54 & ilekudinoside $\mathrm{O}$ & $\mathrm{E}$ & $\mathrm{S} 10$ & - & - & - & - & [20] \\
\hline 55 & ilekudinoside P & $\mathrm{E}$ & S5 & - & - & - & - & [20] \\
\hline 56 & ilekudinchoside C & $\mathrm{E}$ & S8 & - & - & - & - & [27] \\
\hline 57 & ilekudinchoside D & $\mathrm{E}$ & S2 & - & - & - & - & [27] \\
\hline 58 & $\beta$-kudinlactone & $\mathrm{F}$ & $\mathrm{H}$ & $\mathrm{OH}$ & $\mathrm{H}$ & - & - & [14] \\
\hline 59 & kudinoside B & $\mathrm{F}$ & S6 & $\mathrm{OH}$ & $\mathrm{H}$ & - & - & [12] \\
\hline 60 & kudinoside I (2) & $\mathrm{F}$ & Ara & $\mathrm{OH}$ & $\mathrm{H}$ & - & - & [14] \\
\hline 61 & ilekudinoside J & $\mathrm{F}$ & S7 & $\mathrm{OH}$ & $\mathrm{H}$ & - & - & {$[6]$} \\
\hline 62 & ilekudinoside Q & $\mathrm{F}$ & S1 & $\mathrm{OH}$ & $\mathrm{H}$ & - & - & [20] \\
\hline 63 & kudinoside A (ilekudinchoside A) & $\mathrm{F}$ & S3 & $\mathrm{OH}$ & $\mathrm{H}$ & - & - & $\begin{array}{l}{[12,} \\
27]\end{array}$ \\
\hline 64 & kudinoside C & $\mathrm{F}$ & S4 & $\mathrm{OH}$ & $\mathrm{H}$ & - & - & [12] \\
\hline 65 & ilekudinoside I & $\mathrm{F}$ & $\mathrm{S} 10$ & $\mathrm{OH}$ & $\mathrm{H}$ & - & - & {$[6]$} \\
\hline 66 & ilekudinoside L & $\mathrm{F}$ & S5 & $\mathrm{OH}$ & $\mathrm{H}$ & - & - & [20] \\
\hline 67 & ilekudinoside R & $\mathrm{F}$ & $\mathrm{S} 2$ & $\mathrm{OH}$ & $\mathrm{H}$ & - & - & [20] \\
\hline 68 & 3-O- $\beta$-D-glucopyranosyl $-\beta$-kudinlactone & $\mathrm{F}$ & Glc & $\mathrm{OH}$ & $\mathrm{H}$ & - & - & [24] \\
\hline 69 & ilekudinoside U & $\mathrm{F}$ & S3 & EtO & $\mathrm{H}$ & - & - & [21] \\
\hline 70 & ilekudinchoside B & $\mathrm{F}$ & S3 & $\mathrm{H}$ & $\mathrm{MeO}$ & - & - & [27] \\
\hline 71 & ilekudinchoside F & $\mathrm{F}$ & S3 & $\mathrm{H}$ & EtO & - & - & [28] \\
\hline 72 & ilekudinchoside G & $\mathrm{F}$ & S2 & $\mathrm{H}$ & $\mathrm{MeO}$ & - & - & [28] \\
\hline 73 & kudinoside LZ2 & $\mathrm{F}$ & S3 & \multicolumn{2}{|c|}{$\mathrm{O}$} & - & - & [25] \\
\hline 74 & kudinoside F & G & S3 & $\mathrm{OH}$ & $\mathrm{H}$ & - & - & [14] \\
\hline 75 & ilekudinoside $\mathrm{H}$ & G & S4 & $\mathrm{OH}$ & $\mathrm{H}$ & - & - & {$[6]$} \\
\hline 76 & ilekudinoside M & G & S2 & $\mathrm{OH}$ & $\mathrm{H}$ & - & - & [20] \\
\hline 77 & ilekudinoside S & G & $\mathrm{S} 1$ & $\mathrm{OH}$ & $\mathrm{H}$ & - & - & [20] \\
\hline 78 & ilekudinoside V & G & S3 & \multicolumn{2}{|c|}{$\mathrm{O}$} & - & - & [21] \\
\hline 79 & ilekudinoside G & $\mathrm{H}$ & S4 & - & - & - & - & {$[6]$} \\
\hline 80 & ilekudinoside T & $\mathrm{H}$ & S3 & - & - & - & - & [21] \\
\hline
\end{tabular}

Fig. 1 continued 


\begin{tabular}{|c|c|c|c|c|c|c|c|c|}
\hline 81 & lupeol & I & $\mathrm{Me}$ & $\mathrm{Me}$ & $\mathrm{H}$ & - & - & [22] \\
\hline 82 & betulinic acid & I & $\mathrm{Me}$ & $\mathrm{COOH}$ & $\mathrm{H}$ & - & - & [22] \\
\hline 83 & $3 \beta$-hydroxyl-lup-20(29)-24-methyl ester & I & COOMe & $\mathrm{Me}$ & $\mathrm{H}$ & - & - & [16] \\
\hline 84 & $3 \beta$-palmitoyl-lup-20(29)-24-methyl ester & I & COOMe & $\mathrm{Me}$ & $\begin{array}{c}\mathrm{CH}_{3}\left(\mathrm{CH}_{2}\right. \\
)_{14} \mathrm{CO}\end{array}$ & - & - & [16] \\
\hline 85 & ilekudinol C & I & $\mathrm{CH}_{2} \mathrm{OH}$ & $\mathrm{CH}_{2} \mathrm{OH}$ & $\mathrm{H}$ & - & - & [5] \\
\hline 86 & lup-20(29)-ene-3 $\beta, 24$-diol & I & $\mathrm{CH}_{2} \mathrm{OH}$ & $\mathrm{Me}$ & $\mathrm{H}$ & - & - & [31] \\
\hline 87 & betulin & I & $\mathrm{Me}$ & $\mathrm{CH}_{2} \mathrm{OH}$ & $\mathrm{H}$ & - & - & [22] \\
\hline 88 & 13,28-epoxy- $\alpha$-amyrin & $\mathrm{J}$ & $\mathrm{H}$ & $\mathrm{Me}$ & $\mathrm{Me}$ & & & [5] \\
\hline 89 & ilekudinol A & $\mathrm{J}$ & $\mathrm{OH}$ & \multicolumn{2}{|c|}{$\mathrm{CH}_{2}$} & - & - & [5] \\
\hline 90 & rutin & $\mathrm{K}$ & S9 & $\mathrm{OH}$ & - & - & - & [61] \\
\hline 91 & quercetin & $\mathrm{K}$ & $\mathrm{OH}$ & $\mathrm{OH}$ & - & - & - & [61] \\
\hline 92 & kaempferol & $\mathrm{K}$ & $\mathrm{OH}$ & $\mathrm{H}$ & - & - & - & [61] \\
\hline 93 & 3-CQA (3-O-caffeoylquinic acid) & $\mathrm{L}$ & $\mathrm{H}$ & Caffeoyl & $\mathrm{H}$ & $\mathrm{H}$ & - & {$[30]$} \\
\hline 94 & 4-CQA & $\mathrm{L}$ & $\mathrm{H}$ & $\mathrm{H}$ & Caffeoyl & $\mathrm{H}$ & - & [30] \\
\hline 95 & 5-CQA & $\mathrm{L}$ & $\mathrm{H}$ & $\mathrm{H}$ & $\mathrm{H}$ & Caffeoyl & - & [30] \\
\hline 96 & 3,4-diCQA & $\mathrm{L}$ & $\mathrm{H}$ & Caffeoyl & Caffeoyl & $\mathrm{H}$ & - & [30] \\
\hline 97 & 3,5-diCQA & $\mathrm{L}$ & $\mathrm{H}$ & Caffeoyl & $\mathrm{H}$ & Caffeoyl & - & [30] \\
\hline 98 & 4,5-diCQA & $\mathrm{L}$ & $\mathrm{H}$ & $\mathrm{H}$ & Caffeoyl & Caffeoyl & - & [30] \\
\hline 99 & 4-CQA methyl ester & $\mathrm{L}$ & $\mathrm{Me}$ & $\mathrm{H}$ & Caffeoyl & $\mathrm{H}$ & - & [30] \\
\hline 100 & 3,4-diCQA methyl ester & $\mathrm{L}$ & $\mathrm{Me}$ & Caffeoyl & Caffeoyl & $\mathrm{H}$ & - & [30] \\
\hline 101 & 3,5-diCQA methyl ester & $\mathrm{L}$ & $\mathrm{Me}$ & Caffeoyl & $\mathrm{H}$ & Caffeoyl & - & [30] \\
\hline 102 & 4,5-diCQA methyl ester & $\mathrm{L}$ & $\mathrm{Me}$ & $\mathrm{H}$ & Caffeoyl & Caffeoyl & - & [30] \\
\hline 103 & 3,5-Di-O-caffeoyl epi-quinic acid butyl ester & $\mathrm{L}$ & Butyl & Caffeoyl & $\mathrm{H}$ & Caffeoyl & - & [30] \\
\hline No & Compound name & ref & No & Compound nam & & & & Ref \\
\hline 104 & ilekudinol B & 5 & 115 & ilekudinchoside & & & & [29] \\
\hline 105 & ilelic acid A & 51 & 116 & CA methyl ester & & & & [30] \\
\hline 106 & ilelic acid B & 51 & 117 & caffeic acid & & & & [30] \\
\hline 107 & latifoloside $\mathrm{N}$ & 18 & 118 & cis -roseoside & & & & [18] \\
\hline 108 & latifoloside M & 18 & 119 & Epicatechin (EC) & & & & [61] \\
\hline 109 & ilelic acid C & 51 & 120 & Epigallocatechin & gallate (EGC & & & [61] \\
\hline 110 & ilelic acid D & 51 & 121 & Epicatechin galla & e (ECG) & & & [61] \\
\hline 111 & 3-palmitoyl-11-carbonyl-urs-12-ene & 16 & 122 & mannitol & & & & [31] \\
\hline 112 & ilekudinoside F & 6 & 123 & vanillin & & & & [26] \\
\hline 113 & kudinchagenin I & 13 & 124 & gallic acid & & & & [26] \\
\hline 114 & ulmoidol & 5 & 125 & ferulic acid & & & & [26] \\
\hline
\end{tabular}

Fig. 1 continued

types of medicines to treat arteriosclerosis and obesity. Compounds 85, 89, 104, 114, and 20-22 isolated from I. kudingcha showed potent inhibitory activity in the ACAT assay. Compounds $104\left(\mathrm{IC}_{50} 0.044 \mathrm{mM}\right), \mathbf{2 0}\left(\mathrm{IC}_{50}\right.$ $0.064 \mathrm{mM})$, and $21\left(\mathrm{IC}_{50} 0.073 \mathrm{mM}\right)$ showed higher inhibitory activity than the other compounds $\left(\mathrm{IC}_{50}\right.$
0.154-0.468 mM) and those of lignans ( $\mathrm{IC}_{50} 25-207 \mathrm{mM}$ ) isolated from Schisandra chinensis, Machilus thunbergii, Magnolia denudate, Magnolia ovate, and the polyacetylenes $\left(\mathrm{IC}_{50} 42-86 \mathrm{mM}\right)$ from Panax ginseng [5].

Seventeen triterpenoids isolated from the aqueous extract of $I$. kudingcha were examined for inhibitory 


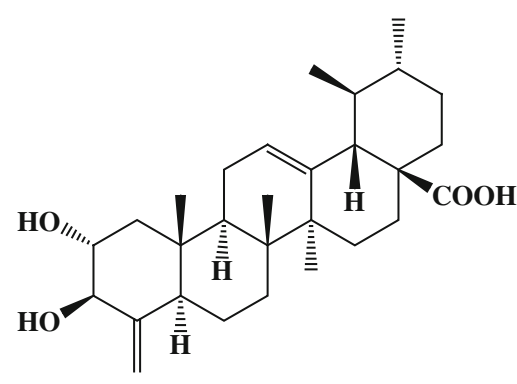

104

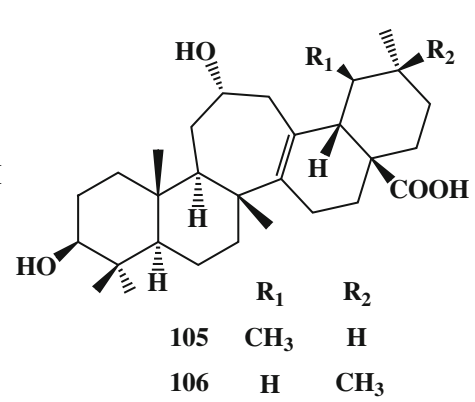

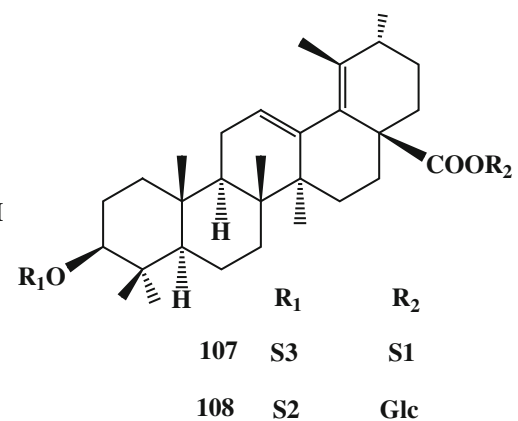

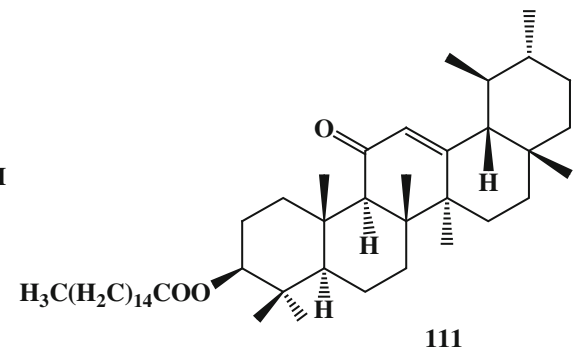

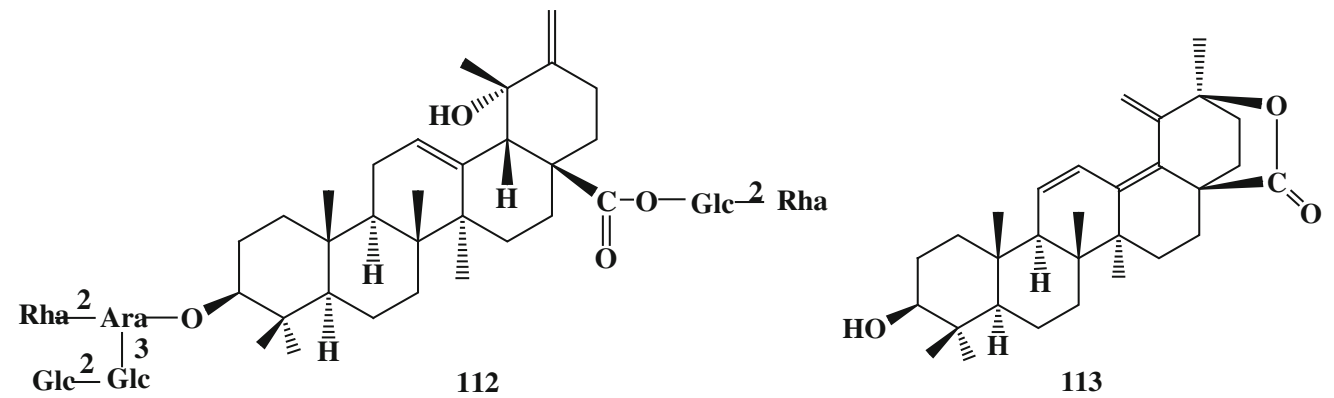

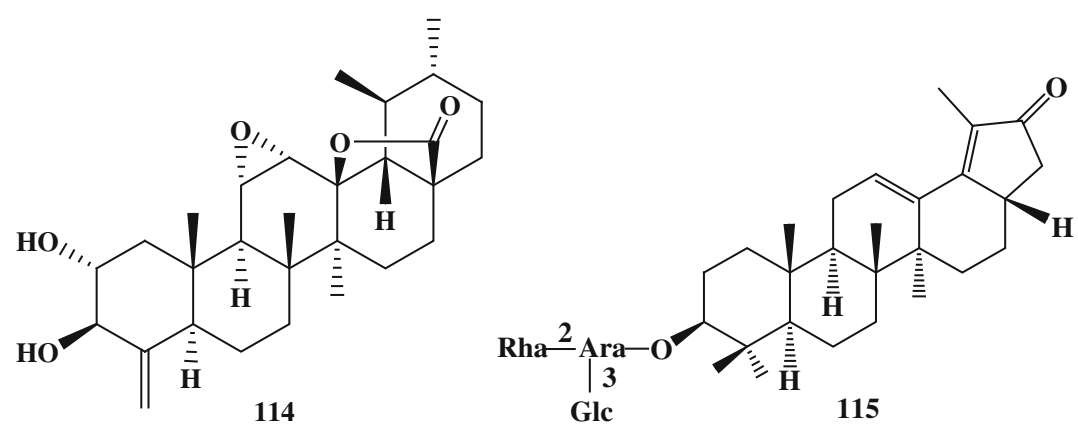

Fig. 1 continued

activity in the ACAT assay. Compounds 4 (the inhibitory activities were $64.3,36.5,15.7 \%$ at $1.0,0.2,0.05 \mathrm{mg} / \mathrm{mL}$, respectively) and $\mathbf{3 6}$ (the inhibitory activities were 63.9, $29.0,31.7 \%$ at $1.0,0.2,0.05 \mathrm{mg} / \mathrm{mL}$, respectively) showed clear inhibitory activity [6].

A cell-based screening model using THP-1 macrophages was initially applied to aggregate LDL (aggLDL)-induced lipid deposition on macrophages to test the inhibitory effects of the 12 triterpenoids from I. kudingcha.
Compounds 63, 64, 49-53, 55, and 62 inhibited the formation of foam cells and reduced intracellular total cholesterol and triglyceride contents. The $\delta$-lactone ring in the aglycone was indispensable to the biological activity. The $\mathrm{OH}$ group at the $\mathrm{C}-12$ position, the number of monosaccharides in the sugar chain, and the presence of a terminal rhamnose residue in the sugar chain might improve this inhibitory effect. Moreover, compound 64 was considered as a potential candidate for the treatment of arteriosclerosis [53]. 
Medium and high doses of total saponins (150 and $300 \mathrm{mg} \mathrm{kg}^{-1}$ day $^{-1}$, respectively) from I. kudingcha reduced the level of serum total cholesterol (TC) in ApoEknockout mice (30-35\%), a reduction that was similar to the group treated with atorvastatin. It was likely that the saponins reduced the level of TC through the inhibition of ACAT activity. In addition, Kudingcha treatment led to greater inhibition of malondialdehyde (MDA) activity than atorvastatin $[6,36]$.

\section{Protection of the vascular system}

Various Ilex species were used in Chinese folk medicine to treat coronary heart diseases. The mode of action was considered to be mediated by their coronary vasodilative effects. The water extract of I. latifolia was shown to increase the contractility and decrease the frequency of contraction in an isolated rat heart perfusion system. The extract inhibited $\mathrm{Na}^{+} / \mathrm{K}^{+}$-ATPase activities in rat heart sarcolemma, rat brain microsome, and a purified enzyme from porcine cerebral cortex. It also inhibited $\mathrm{Ca}^{2+}$ dependent ATPase at a similar dose. Following exposure of the isolated rat heart to the extract at a dose that produces pronounced cardiac effects, the inhibition of $\mathrm{Na}^{+} / \mathrm{K}^{+}$-ATPase activity can be readily detected in the heart [7].

The water extract of I. kudingcha was able to increase coronary blood flow in isolated guinea pig heart and increase cerebral blood flow in anesthetized rabbits, prolong the survival time of mice under hypoxia, and protect rats from myocardial ischemia induced by pituitrin. The effects were beneficial in the prevention and treatment of coronary artery disease and angina pectoris [37].

Intravenous injection $(0.05 \mathrm{~g} / \mathrm{kg})$ of the $I$. kudingcha extract remarkably lowered the blood pressure of normal anesthetized dogs. This hypotensive effect was also seen when the extracts $(0.75$ and $0.94 \mathrm{~g} / \mathrm{kg}$, respectively) were administered to 2-kidney, 1-clip hypertensive rats and spontaneously hypertensive rats by gastric gavage. The subcutaneous abdominal fatty tissue of normal and obese rats decreased significantly when the animals were given the extract $(1.5 \mathrm{~g} / \mathrm{kg})$ by gastric gavage. The results suggested that I. kudingcha had potential as a resource for the treatment of hypertension and obesity [38].

\section{Hypoglycemic effect}

Caco- 2 cells are derived from a human colon adenocarcinoma and are used widely as a model of intestinal absorption by epithelial cells and to study the absorption of glucose in vitro. Glucose absorption by Caco- 2 cells was significantly inhibited by the aqueous extract of $I$. kudingcha under both $\mathrm{Na}^{+}$-dependent conditions and $\mathrm{Na}^{+}$-free conditions, indicating an effect on SGLT1 and GLUT transporters. Analysis of the polyphenols in the extracts suggested that dicaffeoylquinic acids and flavanols may be particularly important in producing these effects. I. kudingcha was the most effective compared to the other plants tested, suggesting that it may merit evaluation in a clinical setting [39].

The water extract of I. latifolia showed marked inhibition of hyperglycemia in the epinephrine hyperglycemia rat model. The blood sugar levels of rats given either a low dose or a high dose of the extract ( 5 or $10 \mathrm{~g} / \mathrm{kg}$, respectively) were significantly reduced compared to the control group, indicating that I. latifolia has potential as a hypoglycemic drug [40].

\section{Antioxidant activity}

The antioxidant activities of $I$. kudingcha have been evaluated in vitro. The crude extract (CE), as well as the 4 fractions of chloroform (CfF), ethyl acetate (EaF), $n$-butanol (nBF), and water (WtF), were evaluated using the 2,2-diphenyl-1-picrylhydrazyl (DPPH) free radicalscavenging assay, Trolox equivalent antioxidant capacity (TEAC), and ferric ion reducing antioxidant power (FRAP). The activities decreased in the order of $\mathrm{EaF}>\mathrm{nBF}>\mathrm{CE}>\mathrm{WtF}>\mathrm{CfF}$, according to the DPPH and FRAP assays, with the exception of the rank order of $\mathrm{CfF}$ and $\mathrm{WtF}$ in the TEAC assay. The extracts were certified to contain a large number of caffeoylquinic acids (CQAs) (93-98), which contributed to the antioxidant activity. From a health point of view, Kudingcha is a beneficial herbal drink due to its antioxidant activity [32].

The ethyl acetate-soluble fraction of I. kudingcha, which contained an abundance of phenolic compounds, displayed remarkable free radical-scavenging activities against DPPH $\left(\mathrm{IC}_{50} 16.3 \mu \mathrm{g} / \mathrm{mL}\right), \quad \mathrm{OH} \quad\left(\mathrm{IC}_{50} 87.5 \mu \mathrm{g} / \mathrm{mL}\right.$ for nonsite-specific and $27.3 \mu \mathrm{g} / \mathrm{mL}$ for site-specific assays) and $\mathrm{O}_{2}{ }^{-}\left(\mathrm{IC}_{50} 1.3 \mu \mathrm{g} / \mathrm{mL}\right)$. The fraction also showed a strong suppressive effect on rat liver mitochondrial peroxidation $\left(\mathrm{IC}_{50} 7.1 \mu \mathrm{g} / \mathrm{mL}\right.$ ) and significantly protected against the oxidation of LDL mediated by either $\mathrm{Cu}^{2+}$ or AAPH free radicals ( $\mathrm{IC}_{50}$ were 14 and $4.8 \mu \mathrm{g} / \mathrm{mL}$, respectively) [30].

The crude polysaccharide KPS II of I. kudingcha and 2 refined polysaccharides, KPS IIIa and KPS IIIb from KPS II, have concentration-dependent hydroxyl radical-scavenging activity. KPS IIIa and KPS IIIb were both composed of arabinose, xylose, mannose, galactose, and glucose, with molar ratios of 10.2:1.0:1.2:8.0:5.4 and 17.7:1.0:1.1:1.8:4.1, respectively. The scavenging capacity of the crude polysaccharide was higher than that of the refined polysaccharides [41].

The contents of total polyphenols and flavonoids of I. latifolia and I. kudingcha were detected by DPPH assay, 
$\mathrm{ABTS}^{+}$assay, OH-free radical-scavenging assay, reducing power assay, and FRAP assay, and were found to be correlated with their antioxidant activities. It was found that the water extract of I. kudingcha had both higher polyphenol content and better antioxidant performance than that of I. latifolia [42].

\section{Antimicrobial activity}

The studies of the anti-bacterial effect of I. latifolia showed an inhibitory effect on the growth of Streptococcus A [minimum inhibitory concentration (MIC) $1,215 \mathrm{mg} / \mathrm{mL}$ ), Pneumococcus (MIC $25 \mathrm{mg} / \mathrm{mL}$ ), Streptococcus B (MIC $25 \mathrm{mg} / \mathrm{mL}$ ), Bacillus diphtheria (MIC $50 \mathrm{mg} / \mathrm{mL}$ ), Bacillus dysenteriae (MIC $50 \mathrm{mg} / \mathrm{mL}$ ), Staphylococcus aureus (MIC $100 \mathrm{mg} / \mathrm{mL}$ ), Pseudomonas aeruginosa (MIC $100 \mathrm{mg} / \mathrm{mL}$ ), Escherichia coli (MIC $100 \mathrm{mg} / \mathrm{mL}$ ), and Micrococcus catarrhalis (MIC $200 \mathrm{mg} / \mathrm{mL}$ ) by the agar dilution method in vitro. Intragastric administration can significantly improve the survival rate of mice infected by E. coli, B. dysenteriae, Pneumobacillus, and Streptococcus B [43].

The crude polysaccharides of I. kudingcha were tested against 7 bacterial and fungal strains commonly known to cause food spoilage, and the results showed that the polysaccharides had more significant activities against bacteria than fungi. The MIC against $S$. aureus was $5 \mathrm{mg} / \mathrm{mL}$ [44].

I. kudingcha was reported to have strong anti-bacterial activity against $S$. aureus, Salmonella typhosa, and $\beta$-hemolytic streptococci, as seen by the agar diffusion method. It could also enhance the action of hypoxia tolerance, hypothermia tolerance, and sports tolerance in the mouse, where it was shown to have anti-stress activities [45].

\section{Antiviral activity}

The anti-HSV-1 activities of different extracts of I. kudingcha were tested by cytopathogenic effect (CPE) observation and plaque reduction assay. The results showed that the aqueous extract had a more pronounced anti-HSV-1 effect $\left(\mathrm{IC}_{50} 108.24 \mu \mathrm{g} / \mathrm{mL}\right.$ ) and lower cytotoxicity (nontoxic concentration $500 \mu \mathrm{g} / \mathrm{mL}$ ) than the ethyl acetate and methanol extract. It is suggested that the extract acts by binding to the HSV-1 receptor and prevents the virus from entering the cells [46].

Studies were also conducted to measure the antiviral effects of I. kudingcha water extract against Coxsackie virus B3 (CVB3) cytotoxicity in HeLa cells by CPE and MTT methods. The results showed that this extract had a significant anti-CVB3 effect in vitro, yielding $\mathrm{TC}_{50}$ values of $45700.3 \mu \mathrm{g} / \mathrm{mL}, \mathrm{IC}_{50}$ values of $119.18 \mu \mathrm{g} / \mathrm{mL}$, and TI of 383.46 in HeLa cells [47].
Enhanced immunity

Aqueous extracts of I. latifolia were found to have beneficial effects on humoral and cellular immunity. The samples enhanced the phagocyte function of macrophages in the mouse abdominal cavity and increased the number of plaque-forming cells (PFCs), indicating that I. latifolia had potency as a new medicine to adjust and enhance the immune function of an organism [48].

To further study this effect, mice were immunocompromised by intragastric treatment with dexamethasone (DEX) for 7 days. The results showed that I. latifolia could improve the phagocytic index of macrophages, and high doses could distinctly enhance the number of PFCs and the percentage of phagocytic macrophages [49].

\section{Anti-tumor activity}

The anti-tumor activity of the ethanol extract and the volatile oils of the old leaves and fresh leaves of I. latifolia were tested by MTT assays. The results showed that the ethanol extract from the old leaves and the volatile oil from the fresh leaves had the most potent anti-tumor activity. For the ethanol extract of old leaves, the inhibition rate against human gastric cancer cells SGC-7901 was $62.40 \%$. For the volatile oil from the fresh leaves, the inhibition rates against human lung cancer cells NCI-H460 ( $\mathrm{IC}_{50} 42.86 \mathrm{mg} / \mathrm{mL}$ ) and SGC-7901 were 93.33 and $32.44 \%$, respectively. The results provided a scientific basis for the use of the tea in the development of new anti-tumor medicines [50].

The growth inhibitory activities of compounds 105,106 , 109, 110, 21, and 22 were evaluated in MCF-7 (estrogen receptor-positive) and MDA-MB-231 (estrogen receptornegative) human breast cancer cells. Compounds $\mathbf{1 0 5}$ and 106 showed a growth inhibitory effect against MCF-7 cells, with $\mathrm{IC}_{50}$ values of $29.51 \pm 3.44$ and $38.49 \pm 3.16 \mu \mathrm{M}$, respectively. Compound $21\left(\mathrm{IC}_{50} 4.58 \pm 0.56 \mu \mathrm{M}\right)$ was the most potent among all the tested compounds, indicating that the $p-(E)$-coumaroyl moiety at the $\mathrm{C}-27$ position may contribute to improving the growth inhibitory potential when compared with the triterpene with the $p$-(Z)-coumaroyl moiety $\left(22, \mathrm{IC}_{50} 12.65 \pm 0.94 \mu \mathrm{M}\right)$. All the tested triterpenoids showed more potent growth inhibitory activity in MCF-7 cells than in MDA-MB-231 cells, implying that their growth inhibitory activities may be partly dependent on the status of the estrogen receptor [51].

The human nasopharyngeal carcinoma cell line NCE was divided into groups A (treated with $40 \mu \mathrm{mol} / \mathrm{L}$ compound 18), B (treated with DMSO $10 \mu \mathrm{L}$ ), and C (without treatment), and the inhibition of cellular proliferation were detected by Western blotting at 12, 24, 48, 72, 96, and $120 \mathrm{~h}$ after treatment. The cellular proliferation in group A was remarkably inhibited at all time points, and the 
inhibitory rate increased from 16.3 to $96.4 \%$. Under an optical microscope, the growth of NCE cells was inhibited, and the morphology changed after treatment. The Western blotting results showed that $\mathbf{1 8}$ could downregulate the expression of ERK and Cyclin D1 in a time-dependent manner. The data suggest that $\mathbf{1 8}$ could be used for treating nasopharyngeal carcinoma by downregulating the expression of ERK and Cyclin D1 in nasopharyngeal carcinoma cells [52]. In another study, an MTT assay was used to detect the effect of $\mathbf{1 8}$ on cellular proliferation, microscopy was used to observe cytotoxicity, and flow cytometry was used to detect apoptosis and cell cycle inhibition. The results showed that, within $24 \mathrm{~h}$ of treatment, 40 and $80 \mu \mathrm{mol} / \mathrm{L}$ of $\mathbf{1 8}$ inhibited the proliferation of NEC-2 cells by $41.21 \pm 0.25$ and $94.11 \pm 0.37 \%$, respectively. Both doses could block more than $70 \%$ of cells at G0/G1. After $24 \mathrm{~h}$ of treatment with $\mathbf{1 8}(40 \mu \mathrm{mol} / \mathrm{L})$, cell atrophy appeared, cell adhesion decreased, and some cells died from fragmentation [64].

Protective activity in neuronal cells

I. latifolia has been used in Chinese folk medicine to treat headaches and various inflammatory diseases. The protective activity of I. latifolia against glutamate-induced neurotoxicity was tested using cultured rat cortical neurons in order to understand the mechanism of its inhibitory effect on ischemic brain damage, and several potentially active compounds were identified. The exposure of cultured cortical neurons to $500 \mu \mathrm{M}$ glutamate for $12 \mathrm{~h}$ triggered neuronal cell death. I. latifolia $(10-100 \mu \mathrm{g} / \mathrm{mL})$ inhibited glutamateinduced neuronal death, elevation of intracellular calcium $\left(\left[\mathrm{Ca}^{2+}\right]_{\mathrm{i}}\right)$, generation of reactive oxygen species (ROS), increase of a pro-apoptotic protein, BAX, and decrease of an anti-apoptotic protein, BcL-2. Hypoxia-induced neuronal cell death was also inhibited by I. latifolia. Compounds $\mathbf{9 8}$, 99, and $\mathbf{1 0 1}$ isolated from I. latifolia also inhibited the glutamate-induced increase in $\left[\mathrm{Ca}^{2+}\right]_{\mathrm{i}}$, generation of ROS, the change of apoptosis-related proteins, and neuronal and hypoxia-induced neuronal cell deaths. The results suggested that I. latifolia and its active compounds prevented glutamate-induced neuronal cell damage by inhibiting the increase of $\left[\mathrm{Ca}^{2+}\right]_{i}$, generation of ROS, and, resultantly, the apoptotic pathway. In addition, the neuroprotective effects on ischemia-induced brain damage might be associated with the anti-excitatory and anti-oxidative actions, and could be attributable to these active compounds, CQAs [54].

Effect on pulmonary symptoms, including cough, asthma, and expectoration

The effects of I. latifolia on isolated guinea pig tracheal smooth muscle cells were evaluated by obtaining $\mathrm{CaCl}_{2}$ and histamine accumulative dose-response curves in vitro. After incubation with the aqueous extract of I. latifolia, the dose-response curves of $\mathrm{CaCl}_{2}$ and histamine were significantly shifted to the right and the maximal contractile force was reduced. The extract could inhibit the isolated tracheal strip contraction induced by acetylcholine and histamine ( $\mathrm{IC}_{50}$ pf 0.16 and $0.21 \mathrm{mg} / \mathrm{mL}$, respectively). It also had a significant dilating effect on tracheal smooth muscle, which indicated that I. latifolia had an important effect on pulmonary symptoms [55].

\section{Toxicity}

Kudingcha has been historically consumed as a functional tea, reflecting the philosophy of medicine-food homology in traditional Chinese medicine, with little or no acute toxicity. Acute and long-term toxicity of I. latifolia was studied in rats using aqueous extracts. The maximum tolerable dose was $168 \mathrm{~g} / \mathrm{kg}$, indicating that there is no acute toxicity. There were also no effects on body weight, hematopoiesis, and biochemical index in the blood of rats intragastrically treated with aqueous extracts at 4.5 , 9, or $18 \mathrm{~g} / \mathrm{kg}$ for 90 days [56].

\section{Clinical usage}

Studies have shown that drinking tea from both I. kudingcha and I. latifolia for 2 months is sufficient to reduce hypertension nearly as well as or better than common hypertension medications such as nifedipine [57, 58]. More importantly, no adverse reaction was observed. These studies suggested that I. latifolia and I. kudingcha have potential use in the treatment of hypertension.

\section{The relationship between the large-leaved Kudingcha and related teas}

Interestingly, the large-leaved Kudingcha from the genus Ilex has many similarities to Yerba maté tea (I. paraguariensis) from South America and the worldwide popular green tea $(C$. sinensis), a member of the Theaceae family [59]. These 3 teas have all been historically used and have shown antioxidant, lipid-lowering, anti-inflammatory, weight-reducing, anti-tumor, and anti-glycation properties $[2,5,6]$. These similarities could be ascribed to their similar phylogeny and chemical compositions.

In order to better understand the relationship among these teas, further comparison of the main chemical constituents of the large-leaved Kudingcha and the other 2 teas have been discussed. Most notably, the common chemical characters in regular tea are caffeine, 
polyphenols, and catechins. Caffeine, which is related to weight reduction, is the highest in Yerba maté tea, medium in the green tea, and could not be found in the largeleaved Kudingcha [60, 61]. Polyphenols with conformed antioxidant activities are also the highest in Yerba maté tea, followed by green tea and large-leaved Kudingcha. The primary polyphenols in the large-leaved Kudingcha are 3,5-di- $O$-caffeoylquinic acid and related dicaffeoylquinic acids, whereas chlorogenic acid is the main polyphenol in Yerba maté tea. Catechins, which have anti-tumor properties, are the highest in green tea, followed by the large-leaved Kudingcha and Yerba maté tea $[32,62,63]$. Moreover, the saponins were thought to be the source of the bitter flavor and one of the important characteristics in the large-leaved Kudingcha and Yerba maté teas. The aroma components in Ilex and green tea also had several similarities.

The long history, similar chemical structure, and common pharmaceutical activities suggest a close genetic relationship among the large-leaved Kudingcha, Yerba maté tea, and green tea. Although the large-leaved Kudingcha is not as popular worldwide, we should pay more attention to its possible benefits and advantages, such as the lack of caffeine that is found in more ordinary teas.

\section{Conclusions}

A large number of phytochemical studies have led to the identification of multiple constituents of the large-leaved Kudingcha that confer properties such as lipid metabolism, vascular system protection, antioxidant, anti-asthma, antitussive, and anti-tumor activities. A critical assessment of the results presented in this review may provide scientific evidence for reasonable utilization of the original plants of the large-leaved Kudingcha and promote further investigation for the development of new herbal medicine and tea products. More pharmacological activities need to be undertaken and relevant mechanisms understood in order to enhance the utilization and quality of Kudingcha species in the future.

Acknowledgments This research was supported by the National Natural Science Foundation of China (no. 81274188).

\section{References}

1. Li L, Xu LJ, Peng Y, He ZD, Shi RB, Xiao PG (2011) Simultaneous determination of five phenylethanoid glycosides in smallleaved Kudingcha from the Ligustrum genus by UPLC/PDA. Food Chem 131:1583-1588

2. Li L, Xu LJ, Peng Y, Shi RB, Xiao PG (2011) Comparison of green tea and four other kind of teas. China J Chin Mater Med $36: 5-10$
3. He ZD, Lau KM, But PPH, Jiang RW, Dong H, Ma SC, Fung KP, Ye WC, Sun HD (2003) Antioxidative glycosides from the leaves of Ligustrum robustum. J Nat Prod 66:851-854

4. Wu ZY, Raven PH, Hong DY (eds) (2008) Flora of China, vol 11. Science Press, Beijing, pp 359-438

5. Nishimura K, Fukuda T, Miyase T, Noguchi H, Chen XM (1999) Activity-guided isolation of triterpenoid acyl CoA cholesteryl acyl transferase (ACAT) inhibitors from Ilex kudincha. J Nat Prod 62:1061-1064

6. Nishimura K, Miyase T, Noguchi H (1999) Triterpenoid saponins from Ilex kudincha. J Nat Prod 62:1128-1133

7. Woo AYH, Jiang JM, Chau CF, Waye MMY, Cheung WT, Kwan HS, Cheng CH (2001) Inotropic and chronotropic actions of Ilex latifolia inhibition of adenosine-5' $5^{\prime}$-triphosphatases as a possible mechanism. Life Sci 68:1259-1270

8. Zhang CK (1994) Survey of Kudingcha original plant and commodity. Chin Herbal Med 17:13-14

9. Li WL, Guo RL, Fu H, Zheng HC (2003) The original plant research about Kudingcha, gonglao leaves and Gougu. Chin Herbal Med 26:595-598

10. Xiang YH, Lu XC (1998) The research of old tea species from Mountain Dalou. Guizhou Sci 16:216-220

11. Li L, Peng Y, Ma GZ, He CF, Feng YX, Lei QF, Xiao PG (2012) Quantitative analysis of five kudinosides in the large-leaved Kudingcha and related species from the genus Ilex by UPLCELSD. Phytochem Anal 23:677-683

12. Ouyang MA, Wang HQ, Chen ZL, Yang CR (1996) Triterpenoid glycosides from Ilex kudincha. Phytochemistry 43:443-445

13. Wen YX, Liang XY, Cheng GR, Wu N, Kang WJ, Zheng QT, Lu $Y$ (1999) Structural identification of Kudinchagenin I. Acta Bot Sin 41(2):206-208

14. Ouyang MA, Yang CR, Chen ZL, Wang HQ (1996) Triterpenes and triterpenoid glycosides from the leaves of Ilex kudincha. Phytochemistry 41:871-877

15. Ouyang MA, Wang HQ, Liu YQ, Yang CR (1997) Triterpenoid saponins from the leaves of Ilex latifolia. Phytochemistry 45:1501-1505

16. Ouyang MA, Wang HQ (1997) Triterpene eaters and triterpenes from Ilex kudingcha. Nat Prod Res Dev 9:19-23

17. Ouyang MA, Liu YQ, Wang HQ, Yang CR (1998) Triterpenoid saponins from Ilex latifolia. Phytochemistry 49:2483-2486

18. Ouyang MA (2001) Glycosides from the leaves of Ilex latifolia. Chin J Chem 19:885-892

19. Ouyang MA, Yang CR, Wu ZJ (2001) Triterpenoid saponins from the leaves of Ilex kudincha. J Asian Nat Prod Res 3:31-42

20. Tang L, Jiang Y, Chang HT, Zhao MB, Tu PF, Cui JR, Wang RQ (2005) Triterpene saponins from the leaves of Ilex kudingcha. J Nat Prod 68:1169-1174

21. Tang L, Jiang Y, Tian XM, Zhou SX, Tu PF (2009) Triterpene saponins from the leaves of Ilex kudingcha. J Asian Nat Prod Res 11:554-561

22. Huang J, Ogihara Y, Shimizu N, Takeda T, Akiyama T (2000) Triterpenoids from stem bark of Ilex latifolia. Nat Med 54:107

23. Huang J, Wang X, Ogihara Y, Shimizu N, Takeda T, Akiyama T (2001) Latifolosides I and J, two new triterpenoid saponins from the bark of Ilex latifolia. Chem Pharm Bull (Tokyo) 49:239-241

24. Che YY, Li N, Zhang L, Tu PF (2011) Triterpenoid saponins from the leaves of Ilex kudingcha. Chin J Nat Med 9:22-25

25. Zuo WJ, Zeng YM, Hu Y, Meng H, Wang ZH, Wang JH (2009) A new triterpene saponin from the leaves of Ilex kudingcha. Chin Chem Lett 20:1331-1334

26. Zuo WJ, Chen HQ, Li XD, Wang ZH, Dai HF, Wang JH (2011) Chemical constituents of Ilex kudingcha leaves. Chin Tradit Herbal Drugs 2:18-20

27. Zuo WJ, Dai HF, Chen J, Chen HQ, Zhao YX, Mei WL, Li X, Wang JH (2011) Triterpenes and triterpenoid saponins from the leaves of Ilex kudincha. Plant Med 77:1835-1840 
28. Zuo WJ, Dai HF, Zeng YB, Wang H, Chen HQ, Wang JH (2012) Two new triterpenoid saponins from the leaves of Ilex kudingcha. J Asian Nat Prod Res 14:308-313

29. Zuo WJ, Wang QH, Li W, Sha Y, Li X, Wang JH (2012) Structure elucidation and NMR assignments of an unusual triterpene saponin derivative from Ilex kudincha. Magn Reson Chem 50:325-328

30. Thuong PT, Su ND, Ngoc TM, Hung TM, Dang NH, Thuan ND, Bae KH, Oh WK (2009) Antioxidant activity and principles of Vietnam bitter tea Ilex kudingcha. Food Chem 113:139-145

31. Liu S, Qin Y, Du FL (2003) Studies on chemical constituents in leafs of Ilex kudingcha. China J Chin Mater Med 28:834-836

32. Liu LX, Sun Y, Laura T, Liang XF, Ye H, Zeng XX (2009) Determination of polyphenolic content and antioxidant activity of Kudingcha made from Ilex kudingcha C.J. Tseng. Food Chem 112:35-41

33. Miho M, Mayumi OK, Tadahiro N (2010) Seasonal difference of caffeic acid derivative contents in current-year leaves and old leaves of Ilex latifolia Thunb. Nippon Shokuseikatsu Gakkaishi 20:305-312

34. Yang XS, Zhao C, Zhou X, Luo B, Yang FM, Hao XJ (2002) The constituents of volatile oil from Ilex kudincha. Acta Bot Yunnan $24: 406-408$

35. Xiong B, Lu HN, Liu L, Xie XM, Deng QY (2003) GC-MS determination of essential oil from Ilex kudingcha C.J. Tseng. J Instrum Anal 22:67-69; Sunyatseni 40:92-94

36. Zheng J, Huang W, Liu GQ, Gu YQ, Tu PF (2009) Total saponins from Kuding tea (Ilex kudingcha C.J.Tseng) protect against kidney injury induced by hypercholesterolemia in apolipoprotein E knockout mice. Chin J New Drugs 18:429-433

37. Zhu LF, Li MZ, Zhong WX, Li AH, Luo JP, Fang ZJ (1994) The cardiovascular pharmacological research on Kudingcha. J Chin Med Mater 17:37-40

38. Chen Y, Li KS, Xie TG (1995) Hypotensive action of the extract of Kudingcha dongqingye (Ilex kudingcha). Chin Tradit Herbal Drugs 26:250-253

39. Wang Z, Clifford MN, Sharp P (2008) Analysis of chlorogenic acids in beverages prepared from Chinese health foods and investigation, in vitro, of effects on glucose absorption in cultured Caco-2 cells. Food Chem 108:369-373

40. Qu LZ, Lu T, Lu PJ, Xi JY (1999) The effect of Kudingcha on epinephrine hyperglycemia rats. Tradit Chin Drug Res Clin Pharmacol 10:279-280

41. Wang X, He LL, Liu B (2008) Study on extraction, purification and scavenging activity to hydroxyl radicals of polysaccharides from leaves of Ilex kudincha C.J. Tseng. Food Sci 29:37-40

42. Zhang WQ, Xu WQ, Sun Y, Ye H, Zeng XX (2010) Comparative evaluation of antioxidant activity in vitro of aqueous extracts from Ilex kudingcha C.J. Tseng and Ilex latifolia Thunb leaf teas. Food Sci 31:22-26

43. Jiang JM, Wang B, Xu SB, Jiang RX (2001) Research on the antibacterial activity of Kudingcha. Pharm Clin Chin Mat Med $17: 18-19$

44. Lin XP, Chen XQ, Su YC, Chen HB (2008) Antimicrobial activities of polysaccharide extracts from Flos lonicerae and Ilex kudingcha. Subtrop Plant Sci 37:51-53

45. Dong Y, Qiao JC, Zhang XL, Liang YH (2001) The experimental study of Ilex latifolia Thunb on pharmacodynamics. J Mudanjiang Univ 22:7-9

46. Hao J, Zhang MY, Wang YF, Wang YF, Wang XY, Pei Y (2008) Study on anti-HSV-1 activity of Ilex kudingcha in vitro. Lishizhen Med Mat Med Res 19:1806-1807

47. Wang YF, Zhang MY, Hao J, Wang XY, Qian CW, Wang YF, Zhang YJ, Yang CR, Kaio K, Nobuyuki K (2008) Study on anti- coxsackie virus effect of aqueous extracts from Ilex kudingcha in vitro. J Tradit Chin Med Pharm 23:976-978

48. Dong Y, Bai XF, Shi XK, Song BH, Zhang XL, Liu YW (2001) The effect of Kudingcha on immune function in mice. J Mudanjiang Univ 22:6-7

49. Sun YB, Wang SQ, Yu XH, Zhang XL, Song BH (2009) Effect of Ilex latifolia Thunb on the regulation of immune function in mice. J Mudanjiang Univ 30:14-16

50. Chen MF, Li XC, Yang SH, Feng YJ, Jiang JH (2007) The antitumor activities of the leaves of Ilex latifolia Thunb. China For Sci Technol 21:30-31

51. Wang CQ, Wang L, Fan CL, Zhang DM, Huang XJ, Jiang RW, Bai LL, Shi JM, Wang Y, Ye WC (2012) Ilelic acids A and B, two unusual triterpenes with a seven-membered ring from Ilex latifolia. Org Lett 14:4102-4105

52. Nong CZ, Huang CJ, Guo LX, Huang ZH, Yang ZG, Wei SY (2010) The effects of ursolic acid of Kuding tea for reducing the proliferation of human nasopharyngeal carcinoma cell line NCE by down-regulating the expressions of ERK and Cyclin D1. Chin Pharmacol Bull 26:1678-1679

53. Zheng J, Tang L, Xian XD, Zhou SX, Shi HM, Jiang Y, Gu YQ, Liu G, Tu PF (2009) Inhibitory effect of triterpenoid saponins from the leaves of Ilex kudingcha on aggregated LDL-induced lipid deposition in macrophages. Plant Med 75:1410-1414

54. Kim JY, Lee HK, Hwang BY, Kim SH, Yoo JK, Seong YH (2012) Neuroprotection of Ilex latifolia and caffeoylquinic acid derivatives against excitotoxic and hypoxic damage of cultured rat cortical neurons. Arch Pharmacal Res 35:1115-1122

55. Jiang JM, Xu SB, Kong YC (2001) The effect of Kudingcha (Ilex latifolia Thunb.) on the contraction of isolated guinea-pig tracheal smooth muscle in vitro. China J Chin Mater Med 26:853-856

56. Xu SB, Jiang JM, Xu DH, Jiang RX, Kong YC, Hu XY (2001) The toxicological studies of the water extraction from Ilex latifolia Thunb. Acta Sci Nat Univ 3:92-94

57. Huang ZC (1997) Observation of hypertension 35 cases treated with Kudingcha. Chin J Inf TCM 4:25

58. Mou LH (2005) Contrast observation on clinical efficacy of Ilex latifolia Kudingcha from Wuyuan Jiangxi on hypertension. Mod Diagn Treat 16:223

59. Heck CI, De Mejia EG (2007) Yerba Mate Tea (Ilex paraguariensis): a comprehensive review on chemistry, health implications, and technological considerations. J Food Sci 72:R138R151

60. Isolabella S, Cogoi L, López P, Anesini C, Ferraro G, Filip R (2010) Study of the bioactive compounds variation during yerba mate (Ilex paraguariensis) processing. Food Chem 122:695-699

61. Liang YR, Ma WY, Lu JL, Wu Y (2001) Comparison of chemical compositions of Ilex latifolia Thumb and Camellia sinensis L. Food Chem 75:339-343

62. Gao YH, Li BQ, Xie CF, Lou HX (2007) Determination of tea polyphenols and caffeine in green tea at different harvest time. Chin J Pharm Anal 27:1790-1793

63. Zaveri NT (2006) Green tea and its polyphenolic catechins: medicinal uses in cancer and noncancer applications. Life Sci 78:2073-2080

64. Nong CZ, Huang CJ, Li JY, Huang ZH, Guo LX, Wei SY, Nong SY (2011) Exploring the proper intervention time and concentration of ursolic acid of Kuding tea on human nasopharyngeal carcinoma cell. Chin J Modern Appl Pharmacy 28:497-500

65. Chen SK (1999) Flora of China, vol 45. Science Press, Beijing

66. Zhang MZ, Qiu LQ (1992) Flora of China, vol 61. Science Press, Beijing, p 61 